\title{
Optoelectronic properties of diketopyrrolopyrrole homopolymers compared to donor-acceptor copolymers
}

Cite as: J. Chem. Phys. 154, 054309 (2021); https://doi.org/10.1063/5.0038284

Submitted: 20 November 2020 • Accepted: 05 January 2021 • Published Online: 04 February 2021

(iD) Ulrike Salzner

\section{COLLECTIONS}

Paper published as part of the special topic on Special Collection in Honor of Women in Chemical Physics and Physical Chemistry
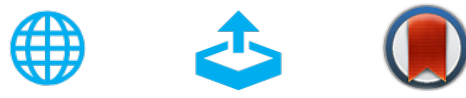

\section{ARTICLES YOU MAY BE INTERESTED IN}

Excitonic and charge transfer interactions in tetracene stacked and T-shaped dimers The Journal of Chemical Physics 154, 044306 (2021); https://doi.org/10.1063/5.0033272

Vibronic coupling in the first six electronic states of pentafluorobenzene radical cation: Radiative emission and nonradiative decay

The Journal of Chemical Physics 154, 054313 (2021); https://doi.org/10.1063/5.0039923

Density-functional thermochemistry. III. The role of exact exchange

The Journal of Chemical Physics 98, 5648 (1993); https://doi.org/10.1063/1.464913

\section{Chemical Physics Reviews}

\section{First Articles Now Online! $=$ READNOW »»}




\title{
Optoelectronic properties of diketopyrrolopyrrole homopolymers compared to donor-acceptor copolymers
}

\author{
Cite as: J. Chem. Phys. 154, 054309 (2021); doi: 10.1063/5.0038284 \\ Submitted: 20 November 2020 - Accepted: 5 January 2021 • \\ Published Online: 4 February 2021
}

Ulrike Salzner $^{\text {a) }}$ (D)

\section{AFFILIATIONS}

Department of Chemistry, Bilkent University, 06800 Bilkent, Ankara, Turkey

Note: This paper is part of the JCP Special Collection in Honor of Women in Chemical Physics and Physical Chemistry.

a) Author to whom correspondence should be addressed: salzner@fen.bilkent.edu.tr

\begin{abstract}
Diketopyrrolopyrrole (DPP) is a component of a large number of materials used for optoelectronic applications. As it is exclusively used in combination with aromatic donors, the properties of its homopolymers are unknown. Because donor-acceptor character has been shown for other systems to reduce bandwidths, DPP homopolymers should have even larger conduction bands and better $n$-type conductivity than the thiophene-flanked systems, which have exceptional $n$-type conductivity and ambipolar character. Therefore, a theoretical study was carried out to elucidate the properties of the unknown DPP homopolymer. Calculations were done with density functional theory and with the complete active space self-consistent field method plus n-electron valence state perturbation theory for the dynamic correlation. Poly-DPP is predicted to have radical character and an extremely wide low-lying conduction band. If it were possible to produce this material, it should have unprecedented $n$-type conductivity and might be a synthetic metal. A comparison with various unknown donor-acceptor systems containing vinyl groups and thienyl rings with a higher concentration of DPP than the known copolymers reveals how donor-acceptor substitution reduces bandwidths and decreases electron affinities.
\end{abstract}

Published under license by AIP Publishing. https://doi.org/10.1063/5.0038284

\section{INTRODUCTION}

Diketopyrrolopyrrole (DPP) based conducting polymers are among the most successful systems for organic optoelectronic devices. ${ }^{1}$ In particular, their high electron affinities (EAs) and wide conduction bands lead to unusually high electron conductivities ${ }^{2}$ and allow for the realization of ambipolar conductors. ${ }^{3}$ Recently, DPP systems were shown to undergo singlet fission ${ }^{4,5}$ and to be good candidates for thermoelectrics.

DPP flanked by two phenyl rings was first reported in the literature as a very stable brightly colored pigment. ${ }^{7}$ Often, the abbreviation DPP is used for the entire system including the aromatic rings. The phenyl rings can be replaced with other aromatic units, for instance, thiophene (T). ${ }^{8,9}$ Polymerization of T-DPP-T affords ambipolar conducting polymers. ${ }^{10,11}$ T-DPP-T-systems have donor-acceptor character, with DPP being the acceptor and thiophene being the donor. The aromatic units can be varied, and additional acceptors may be combined with DPP in order to fine-tune the properties of the polymers. However, the properties of poly-DPP homopolymers seem to be unknown as a literature search for DPP homooligomers or DPP polymers gave no results. Since the known methods for the synthesis of DPP systems yield only species flanked with aromatic donors, a completely different synthetic approach might be necessary. Before starting such an endeavor, it is desirable to establish whether homopolymers of DPP are likely to have any advantage over the readily available donor-acceptor systems.

The donor-acceptor concept was originally conceived with the aim of decreasing bandgaps and simultaneously increasing bandwidths. ${ }^{12}$ Theoretical studies have shown, however, that properties of donor-acceptor systems are between those of the corresponding homopolymers. ${ }^{13-16}$ While bandgaps can indeed be tuned over a wide range, bandwidths decrease with an increasing energy difference between a donor and an acceptor. This is accompanied 
by localization, usually of the conduction band states and a decrease in the intensity of the first band in the absorption spectrum. ${ }^{16}$ The localization of the conduction band leads to a decrease in $n$-type conductivity even if the electron affinity of the system is high. ${ }^{14}$ These findings suggest that DPP homopolymers should have higher electron affinities and wider conduction bands than T-DPP-T donor-acceptor copolymers. Thus, pure DPP polymers could have interesting opto-electronic properties in terms of $n$-type conductivity, ambipolar transport, and stability of n-doped polymers under ambient conditions. To elucidate these possibilities, a detailed theoretical investigation of pure DPP oligomers and of a variety of donor-acceptor systems with a higher DPP content than commonly used was performed. The results regarding these so far unknown systems are indeed promising, and it is hoped that some of these systems will eventually be synthesized and exploited experimentally.

\section{METHODS}

Structures of $\mathbf{D P P}_{\mathrm{n}}$ oligomers with $\mathrm{n}=1-24$, of methyl substituted DPP $\left(\mathbf{D P P M}_{\mathrm{n}}\right)$ with $\mathrm{n}=1-12$, of vinyl and thiophene spaced DDPM $\left(\mathbf{D P P M}-\mathrm{V}_{\mathrm{n}}\right)$ and $\left(\mathbf{D P P M}-\mathbf{T}_{\mathrm{n}}\right)$ with $\mathrm{n}=2-12$, and of bithiophene spaced DPPM (T-DPPM-T ${ }_{n}$ ) with $n=1-7$ (see Scheme 1 for structures of trimers) were optimized with density functional theory (DFT) employing the B3P86 ${ }^{17,18}$ functional with $30 \%{ }^{19,20}$ of Hartree-Fock exchange and the $6-31 G^{* 21}$ basis set. Results with this functional are reliable and compare well with those obtained with range-separated hybrid functionals. ${ }^{20}$ Wavefunction stability was checked for all species. Appropriate closed- and open-shell (broken symmetry) calculations were used for band structure predictions, for singlet-triplet splittings $\left(\Delta \mathrm{E}_{\mathrm{S}-\mathrm{T}}\right)$, and for excited state calculations with time-dependent DFT (TDDFT). The DFT ground state singlet-triplet energy differences were corrected with Yamaguchi's spin projection formula, ${ }^{22}$

$$
\Delta E_{S-T}=2 J_{a b}=\frac{E_{B S}-E_{T}}{\left\langle S^{2}\right\rangle_{T}-\left\langle S^{2}\right\rangle_{B S}},
$$

where $\mathrm{E}_{\mathrm{BS}}$ and $\left\langle S^{2}\right\rangle_{B S}$ are the energy and expectation value of the spin operator of the broken symmetry calculations.

For DPP oligomers, highly spin-contaminated open-shell wavefunctions resulted. Although the expectation values of the spin operator, $\left\langle S^{2}\right\rangle$, are not clearly defined for DFT calculations, the deteriorating values indicate that the open-shell DFT wavefunctions of longer DPP oligomers are problematic. These systems were, therefore, investigated additionally with complete active space selfconsistent field (CASSCF) calculations using the DFT structures. Occupations of natural orbitals ${ }^{23}$ and fractional occupation numbers from finite temperature DFT with a smear temperature of $11000 \mathrm{~K}$ for B3P86 with $30 \% \mathrm{HF}$ exchange ${ }^{24}$ were analyzed to determine which orbitals should be included in the active space. However, the populations with both approaches decrease very gradually without any obvious gap. Including all occupied natural orbitals with occupancies of less than $1.98 \mathrm{e}$ and all unoccupied molecular

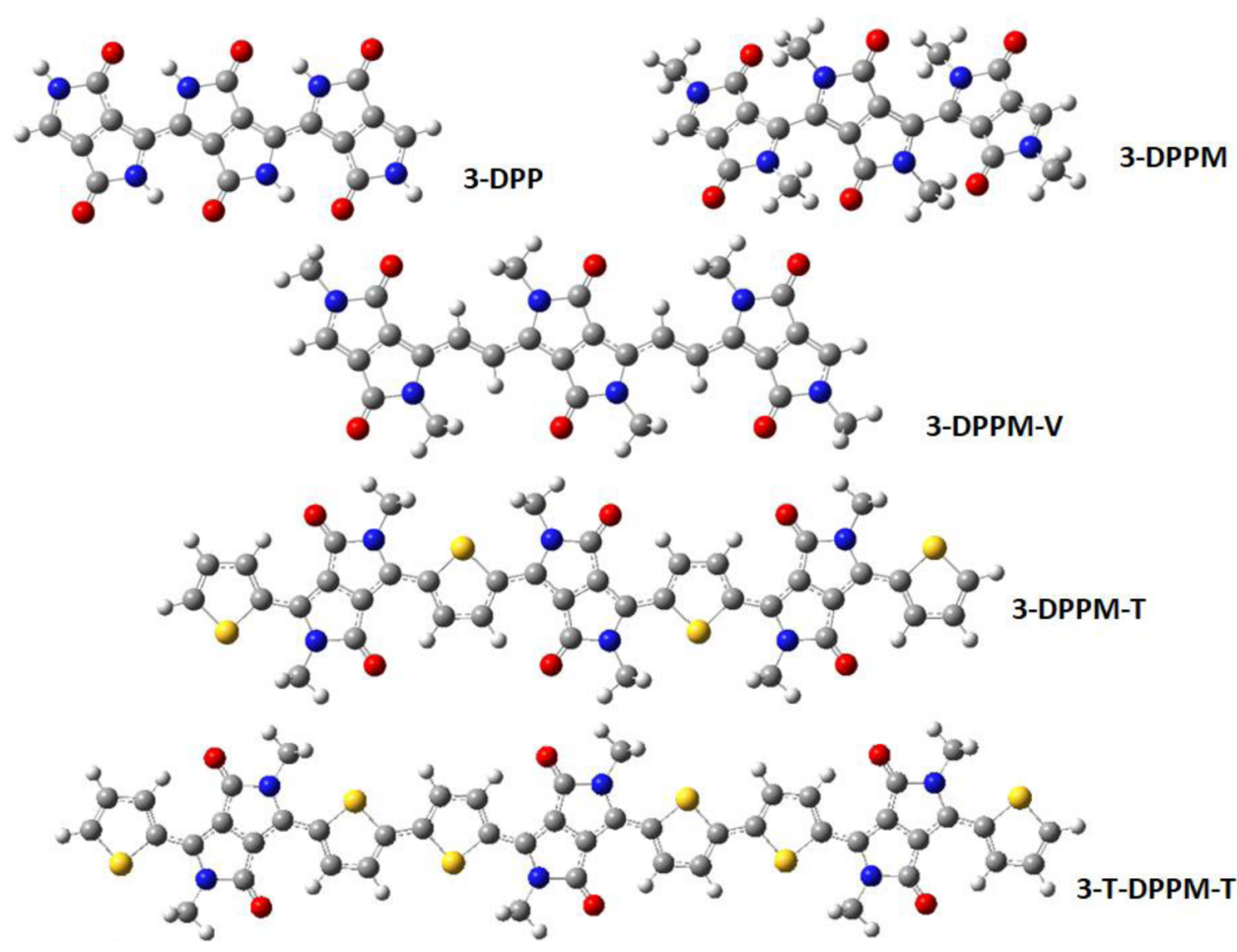

SCHEME 1. Trimers of the repeat units investigated. 
orbitals with more than $0.02 \mathrm{e}$ as suggested in the ORCA man$\mathrm{ual}^{25}$ would lead to huge active spaces including $\sigma$-orbitals. Therefore, two series of test calculations were carried out. The first one used active spaces of four electron and four orbitals [CASSCF $(4,4)]$ for all systems. The longest oligomer that could be treated at this level is 12-DPP. The second series was done with active spaces that include all electrons and orbitals arising from the highest occupied molecular orbitals (HOMOs) and the lowest unoccupied molecular orbitals (LUMOs) of the repeat units. This leads to increasing active spaces $[\operatorname{CASSCF}(2,2)$ for monomers, $\operatorname{CASSCF}(4,4)$ for dimers, and so on] and includes all orbitals that eventually form the valence and conduction bands of the corresponding polymers. (The terms valence and conduction bands will also be used in the following for oligomers.) The longest oligomer that could be handled in this way is 6-DPP with an active space containing 12 electrons and 12 orbitals. 8-DPP would require 16 electrons and 16 orbitals, which is beyond the available computational resources. Finally, more extended active spaces were tested for short oligomers. $\operatorname{CASSCF}(12,12)$ calculations for 3-DPP and 4-DPP showed that the orbital occupancies of the additional low-lying orbitals were significantly higher and those of the additional high lying orbitals were significantly lower than those of the orbitals, forming valence and conduction bands. The excitation energies differed by less than $0.15 \mathrm{eV}$, showing that larger active spaces are not needed.

Dynamic correlation was included via n-electron valence state perturbation theory (NEVPT2). ${ }^{26-28}$ Dynamic correlation is absolutely essential as the excitation energies and the order of the excited states undergo significant changes when dynamic correlation is added. State specific calculations were done for singlet and triplet ground states, and $\Delta \mathrm{E}_{\mathrm{S}-\mathrm{T}}$ were calculated as energy differences. Singlet and triplet excitation energies were calculated with state averaging over three triplet states and the number of singlet states required to include the first strongly allowed singlet state [e.g., nine singlets for 6-DPP with CASSCF $(12,12)]$. The number of singlet states that has to be included is so high because CASSCF without dynamic correlations incorrectly places several dark states below the singlet state. This is especially pronounced with the larger active spaces.

The diradical character (y) of the oligomers was determined from the state averaged CASCF $(4,4)$ NEVPT2 calculations according to the formula of Kamada et al.,

$$
y=1-\sqrt{1-\left(\frac{E 1 S-E 1 T}{E 2 S}\right)^{2}},
$$

where E1S is the energy of the allowed singlet state, E1T is the energy of the first triplet state, and E2S is the energy of the first doubly excited singlet state.

To handle the large systems, the resolution of identity (RI) approximation $^{30}$ for the Coulomb term and the chain of sphere (COSX) approximation ${ }^{31}$ of the exchange term were used. The basis sets were the split valence polarized (SVP) basis sets of the Karlsruhe group ${ }^{32}$ and the auxiliary basis set by Weigend, ${ }^{33}$ which is required for the RI method. The DFT calculations were carried out using Gaussian $16^{34}$ and ORCA. ${ }^{35,36}$ That both program packages lead to the same results was verified. The finite temperature DFT and CASSCF/NEVPT2 calculations were done with ORCA.

\section{RESULTS}

DPP oligomers are planar due to hydrogen bridges between the close lying $\mathrm{N}-\mathrm{H}$ and $\mathrm{C}=\mathrm{O}$ groups. Conjugation along the backbone is extremely strong, leading to interring double bonds and exchange of single and double bond positions in the DPP units compared to the monomer (Scheme 2). The HOMO-LUMO energy gap decreases rapidly. The exchange of single and double bond positions leaves unpaired electrons at the terminal DPP units. As a result, the closed-shell wavefunction becomes unstable for the trimer and the tetramer has already an $\left\langle S^{2}\right\rangle$ value of 1.0. Because DFT calculations might be unreliable for such systems, CASSCF/NEVPT2 calculations were done as well.

In Table I, $\Delta \mathrm{E}_{S-\mathrm{T}}$ from B3P86-30\%/6-31G ${ }^{*}$ and CASSCF/ NEVPT2/SVP ground state calculations and CASSCF/NEVPT2/SVP state averaged excited state calculations are compared. The values with active spaces $(4,4)$ and $(2 n, 2 n)$, with $n$ being the number of repeat units, are reported next to each other. The $\left\langle S^{2}\right\rangle$ values of the DFT calculations deteriorate rapidly for singlet and triplet states starting with 4-DPP. For 8-DPP, one can no longer speak of singlet or triplet states. The DFT $\Delta \mathrm{E}_{\mathrm{S}-\mathrm{T}}$ were, therefore, corrected with Yamaguchi's spin projection formula [Eq. (1)]. ${ }^{22}$ The spin-projected $\Delta \mathrm{E}_{\mathrm{S}-\mathrm{T}}$ decrease from $1.73 \mathrm{eV}$ to $0.41 \mathrm{eV}$ from the monomer to dodecamer and then increase slightly to $0.45 \mathrm{eV}$ for 24-DPP. Without spin projection, $\Delta \mathrm{E}_{\mathrm{S}-\mathrm{T}}$ decreases smoothly to $0.10 \mathrm{eV}$ for the dodecamer and then stays constant up to 24-DPP. The CASSCF/NEVPT2 ground state $\Delta \mathrm{E}_{S-\mathrm{T}}$ also decrease at first, reach a minimum for $4-5$ repeat units, and then increase with both active spaces. This unphysical ${ }^{37}$ chain length dependence occurs at the same chain length where the oligomers develop open-shell character in the DFT calculations. Simultaneously, the ground state CASSCF wavefunction develops significant doubly excited character as the HOMO-LUMO gaps approach 0. Starting with 10-DPP, it was not possible, despite many attempts, to converge to the ground state wavefunction in which the electronic configuration with two doubly occupied lowest energy active orbitals and two unoccupied highest energy active orbitals (2200) has the largest contribution. Instead, (2020) or (2110) "ground states" are obtained for 10-DPP and 12-DPP. This observation can be rationalized by the vanishing HOMO-LUMO gaps.

The CASSCF/NEVPT2 triplet excitation energies decrease monotonically from $2.06 \mathrm{eV}$ to $0 \mathrm{eV}$ from the monomer to decamer and dodecamer. Note, however, that wavefunctions of the latter two are mixtures of many electron configurations. The CASSCF/NEVPT2 calculations reveal, therefore, that $\Delta \mathrm{E}_{S-\mathrm{T}}$ of DPP systems with ten repeat units and more is 0 and that ground and doubly excited states are close in energy. The diradical character y increases from very small values for the closed-shell (according to DFT) oligomers to 0.549 for 8-DPP. For the longer oligomers, E1T approaches zero, and E2S is smaller than E1S. Therefore, the bracket in Eq. (2) is larger than 1, the root argument is negative, and the root becomes imaginary. The value of $y$ is, however, close to 1 , as expected for diradicals.

All DPP oligomers give rise to a strong singlet excited state at low energy. Below lie two triplet states and one dipole forbidden singlet state. A very weakly allowed feature is predicted by DFT at slightly lower energy and with CASSCF/NEVPT2 at slightly higher energy than the strong state. With DFT, the weakly 

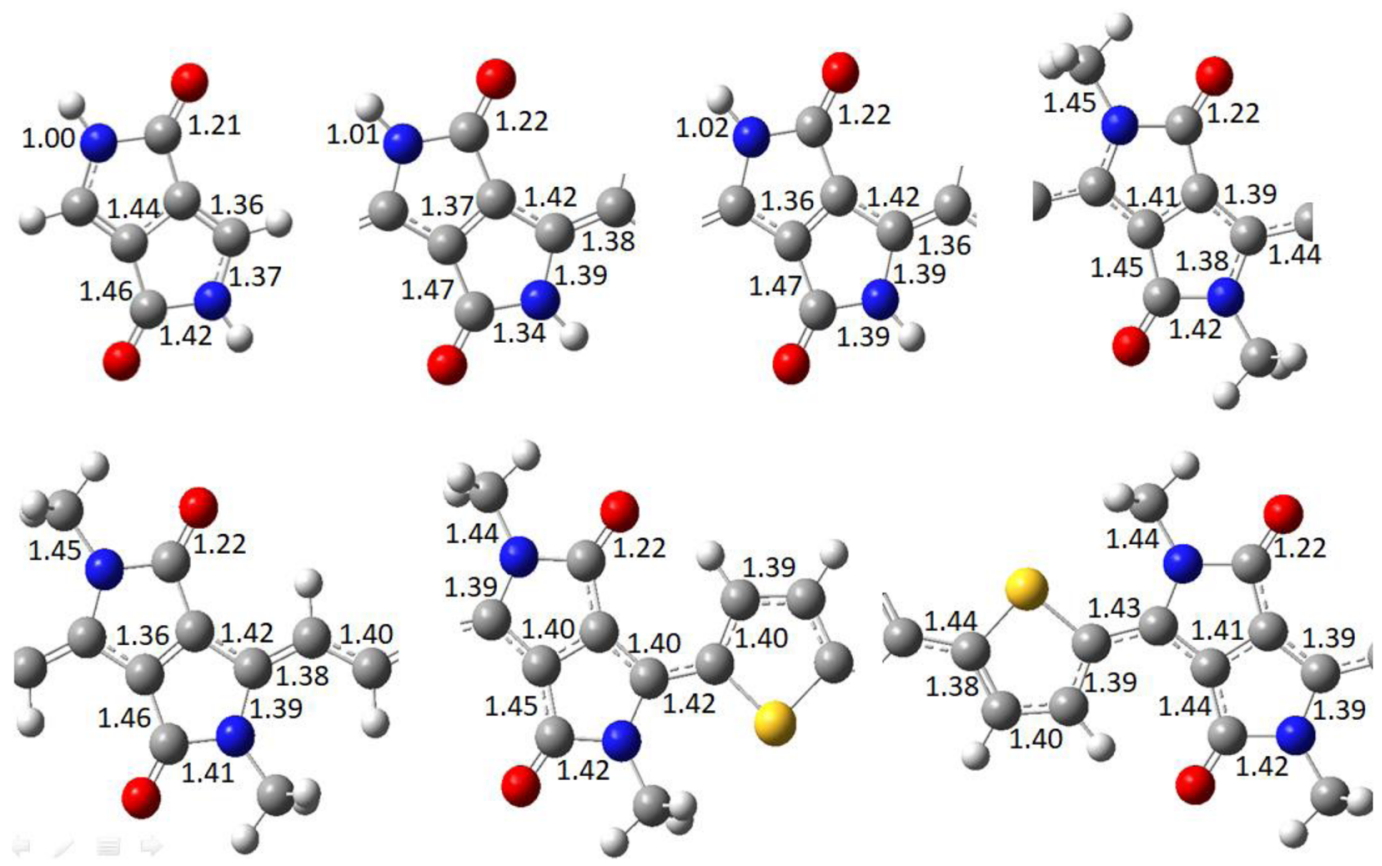

SCHEME 2. Structures of isolated DPP and of the inner rings of 24-DPP, $\mathrm{CH}_{2}$-capped 24-DPP, 12-DPPM, 12-DPPM-V, 12-DPPM-T, and 7-T-DPPM-T.

allowed state is a HOMO-1-LUMO plus HOMO-LUMO+1 transition. With CASSCF/NEVPT2, it is also a HOMO-1-LUMO plus $\mathrm{HOMO}-\mathrm{LUMO}+1$ transition, but there is an additional double excitation character. The strong state is dominated by HOMO-LUMO transitions with both methods, and the absorption energies decrease with chain length. The CASSCF/NEVPT2 values with both active spaces differ by less than $0.1 \mathrm{eV}$ and are lower than the DFT values by $0.03 \mathrm{eV}-0.4 \mathrm{eV}$. Considering the catastrophic spin contamination, DFT excitation energies are remarkably close to the CASSCF/NEVPT2 values. Thus, broken symmetry TDDFT excitation energies are at least qualitatively acceptable. The further comparisons are done with DFT calculations only.

TABLE I. $\left\langle\mathrm{S}^{2}\right\rangle$ values of the B3P86-30\% (DFT) "singlet" and "triplet" wavefunctions, energy difference between spin-projected DFT and CASSCF/NEVPT2 (CAS) ground state $\triangle \mathrm{E}_{\mathrm{S}-\mathrm{T}}$, CASSCF/NEVPT2 triplet excitation energies ( $\left.{ }^{3} \mathrm{exc}\right), \mathrm{B} 3 \mathrm{P} 86-30 \%$ and CASSCF/NEVPT2 singlet excitation energies ( $\left.{ }^{1} \mathrm{exc}\right)$, diradical character y at CAS(4,4), and DFT $\triangle S C F$ IEs and EAs of DPP oligomers in eV.

\begin{tabular}{|c|c|c|c|c|c|c|c|c|c|}
\hline \multirow[b]{3}{*}{ DPP } & \multirow{3}{*}{$\frac{\left\langle\mathrm{S}^{2}\right\rangle \mathrm{S}_{0} / \mathrm{T}_{0}}{0 / 2.0}$} & \multicolumn{2}{|c|}{$\Delta \mathrm{E}_{\mathrm{S}-\mathrm{T}}$} & \multirow{2}{*}{$\frac{{ }^{3} \mathrm{exc}}{(2 n, 2 n)}$} & \multicolumn{2}{|r|}{${ }^{1}$ exc } & \multirow{3}{*}{$\frac{y}{\operatorname{CAS}(4,4)}$} & $\mathrm{IE}$ & \multirow{2}{*}{ EA } \\
\hline & & \multirow{2}{*}{$\begin{array}{c}\text { DFT } \\
1.73\end{array}$} & $\operatorname{CAS}(4,4) /(2 n, 2 n)$ & & \multirow{2}{*}{$\frac{\text { DFT }}{3.65}$} & \multirow{2}{*}{$\frac{\operatorname{CAS}(4,4) /(2 \mathrm{n}, 2 \mathrm{n})}{3.65 / 3.38}$} & & DFT & \\
\hline & & & $2.13 / 2.16$ & $2.30 / 2.06$ & & & & 8.54 & 0.95 \\
\hline 2-DPP & $0 / 2.0$ & 1.01 & 1.59 & 1.34 & 2.55 & 2.49 & 0.086 & 8.06 & 2.65 \\
\hline 3-DPP & $0.4 / 2.1$ & 0.79 & $0.41 / 0.91$ & $1.13 / 1.00$ & 2.11 & $2.04 / 2.02$ & 0.085 & 7.76 & 3.43 \\
\hline 4-DPP & $1.0 / 2.2$ & 0.61 & $0.31 / 0.11$ & $0.57 / 0.63$ & 1.83 & $1.55 / 1.50$ & 0.213 & 7.53 & 3.92 \\
\hline 5-DPP & $1.3 / 2.2$ & 0.52 & $0.15 / 0.18$ & $0.45 / 0.35$ & 1.67 & $1.30 / 1.24$ & 0.282 & 7.42 & 4.18 \\
\hline 6-DPP & $1.5 / 2.3$ & 0.47 & $0.25 / 0.43$ & $0.31 / 0.24$ & 1.56 & $1.09 / 1.18$ & 0.393 & 7.35 & 4.36 \\
\hline 8-DPP & $1.9 / 2.6$ & 0.42 & $0.79 / \ldots$ & $0.10 / \ldots$ & 1.41 & $0.82 / \ldots$ & 0.549 & 7.27 & 4.59 \\
\hline 10-DPP & $2.2 / 2.7$ & 0.41 & $0.00 / \ldots$ & $0.02 / \ldots$ & 1.32 & $/ \ldots$ & & 7.24 & 4.73 \\
\hline 12-DPP & $2.6 / 3.1$ & 0.41 & $0.00 / \ldots$ & $0.00 / \ldots$ & 1.26 & I. & & 7.24 & 4.81 \\
\hline 16-DPP & $3.3 / 4.2$ & 0.43 & I... & $1 \ldots$ & 1.19 & I. & & 7.23 & 4.92 \\
\hline 20-DPP & $4.0 / 4.4$ & 0.44 & I. & I. & & I... & & 7.24 & 4.98 \\
\hline 24-DPP & $4.7 / 5.1$ & 0.45 & /.. & I. & & I. & & 7.25 & 5.01 \\
\hline
\end{tabular}


The DFT ionization energies (IEs) reach constant values at 10DPP and are, therefore, converged. The final value of $7.25 \mathrm{eV}$ is relatively high compared to other conjugated polymers such as polythiophene, for instance, which has an IE of $6.0 \mathrm{eV}$ at the same level of theory. The electron affinities (EAs) converge more slowly but increase between 20-DPP and 24-DPP by only $0.03 \mathrm{eV}$. The predicted gas phase EA of the polymer of over $>5 \mathrm{eV}$ is huge, and the transport gap, IE-EA, ${ }^{38}$ is very small, $2.24 \mathrm{eV}$. The large EA makes DPP oligomers excellent candidates for $n$-type transport under ambient conditions because anions would be stable against oxidation by oxygen and moisture. However, there would most likely be unintentional doping because the EA exceeds $4.5 \mathrm{eV}$.

In Fig. 1, DFT orbital energies of DPP and thiophene oligomers with up to 24 conjugated double bonds along the backbone are compared. In the middle of the graph, orbital energies of the cooligomer 4-T-DPPM-T, which also has 24 conjugated double bonds, are the inserted. DPP oligomers have much higher EAs and IEs than thiophene oligomers. The valence band of
4-T-DPPM-T is close to that of 12-thiophene, and the conduction band lies midway between that of 12-thiophene and 12-DPP and is much narrower. Thus, the high EA and good $n$-type conductivity of poly-T-DPPM-T are inherited from DPP and not a result of donor-acceptor character. The bandgap of 4-T-DPPM-T, $2.0 \mathrm{eV}$, is almost the same as that of 12-DPP, $1.9 \mathrm{eV}$, but much smaller than that of 12-thiophene, $3.0 \mathrm{eV}$. Occupied and unoccupied energy levels are shifted upward compared to 12-DPP so that the copolymer is easier to oxidize but less stable in the reduced form. Therefore, if it were possible to make poly-DPP, they were expected to have a much higher electron mobility and better $n$-type conductivity than poly-T-DPPM-T.

The unpaired electrons that appear at the terminal DPP units can be paired by end-capping the oligomers with double bonded groups, the simplest being $\mathrm{CH}_{2}$. To test the effect of end-capping on the $\left\langle\mathrm{S}^{2}\right\rangle$ value, closed-shell and broken symmetry DFT calculations were carried out for $\mathrm{CH}_{2}$-capped DPP oligomers. Trimer and tetramer are closed-shell species, but the pentamer
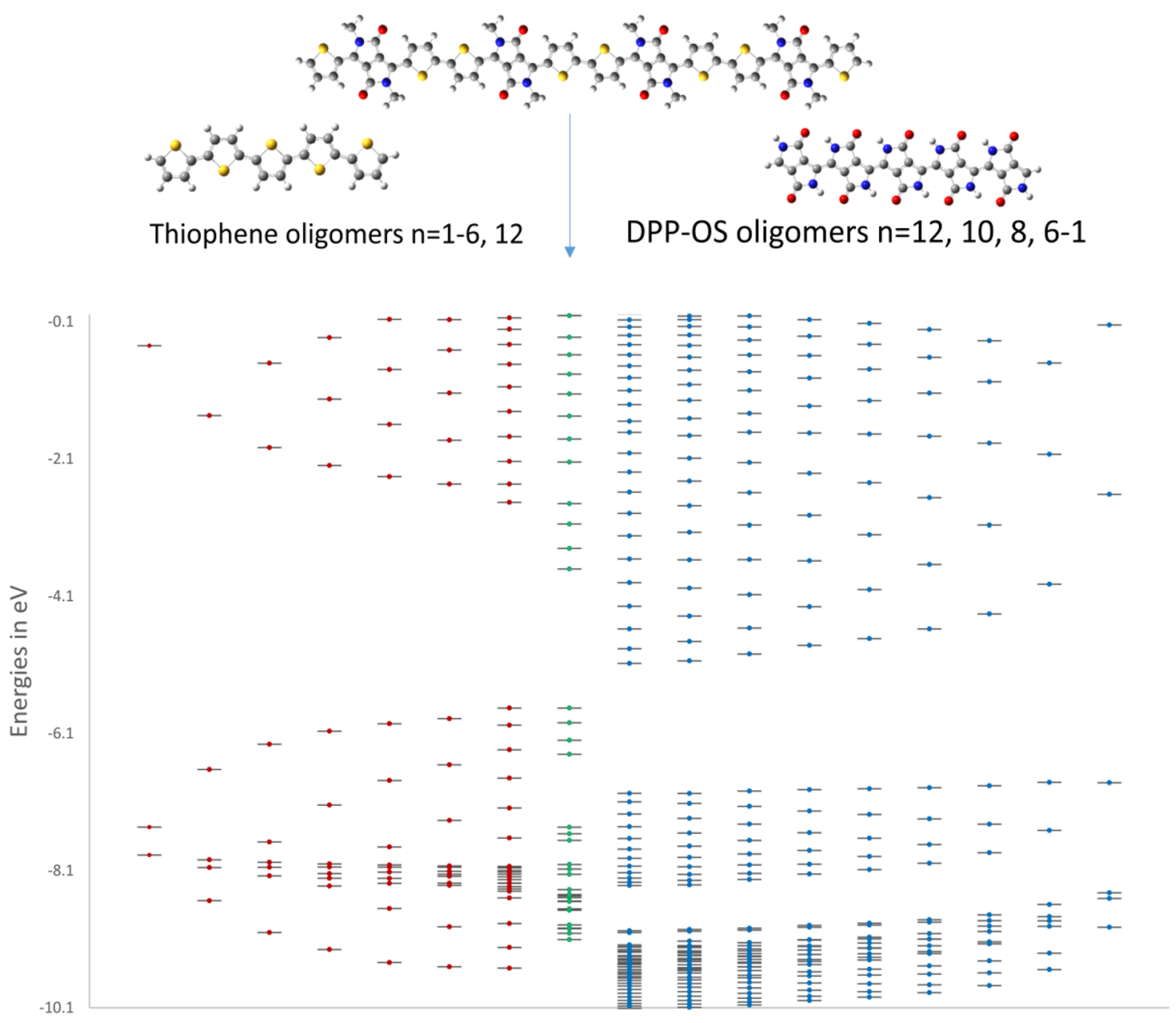

FIG. 1. Orbital energies of thiophene oligomers (left), 4-T-DPPM-T (middle, green markers), and DPP oligomers (right). 
TABLE II. Comparison of negative HOMO and LUMO energies $\left(\mathrm{IP}_{\text {Koop }}\right.$ and $\left.E A_{K o o p}\right)$, bandwidths of valence $\left(B W_{\text {val }}\right)$ and conduction bands ( $\left.B W_{c o n}\right)$, bandgap $\left(E_{g}\right)$, and $E_{S-T}$ in $\mathrm{eV}$, and $\left\langle S^{2}\right\rangle$ of different oligomers with 24 conjugated or close to 24 double bonds ( 23 for DPPM- $V$ and 26 for DPPM-T).

\begin{tabular}{|c|c|c|c|c|c|c|c|}
\hline & $\mathrm{IE}_{\mathrm{Koop}}$ & EA $_{\text {Koop }}$ & $\mathrm{BW}_{\mathrm{val}}$ & $\mathrm{BW}_{\text {con }}$ & $E_{g}$ & $\mathrm{E}_{S-\mathrm{T}}$ & $\left\langle S^{2}\right\rangle$ \\
\hline 12-DPP & 6.97 & 5.07 & 1.34 & 3.36 & 1.90 & 0.41 & 2.59 \\
\hline $\mathrm{CH}_{2}-12-\mathrm{DPP}-\mathrm{CH}_{2}$ & 6.95 & 5.08 & 1.48 & 3.21 & 1.87 & 0.80 & 1.56 \\
\hline 12-DPPM & 6.47 & 4.50 & 1.22 & 2.46 & 1.97 & 0.90 & 1.34 \\
\hline 8-DPPM-V & 6.21 & 4.24 & 1.08 & 2.44 & 1.97 & 0.36 & 2.38 \\
\hline V-8-DPPM-V & 6.17 & 4.23 & 1.08 & 2.28 & 1.94 & 0.36 & 2.46 \\
\hline 6-DPPM-T & 5.77 & 3.97 & 1.08 & 1.69 & 1.80 & 0.81 & 0.74 \\
\hline 4-T-DPPM-T & 5.73 & 3.70 & 0.67 & 0.95 & 2.03 & 1.02 & 0 \\
\hline
\end{tabular}

and hexamer start breaking symmetry. The dodecamer has an $\left\langle S^{2}\right\rangle$ value of 1.6, less than the uncapped dodecamer with $\left\langle S^{2}\right\rangle=2.6$. Hence, end-capping does not make DPP oligomers closed-shell systems but reduces the spin contamination of the wavefunctions considerably. It is interesting to note that the orbital energies differ very little from those of the uncapped isomers. Therefore, end-capping would be a route to increase the stability of pure DPP systems.

Although the data for the DPP oligomers are very promising, there are two issues that might prevent experimental realization. First, the open-shell diradicalic character might render the systems unstable despite their large EA and IE. Second, the planar hydrogenbridged structure indicates that the longer oligomers and polymers are probably insoluble. To address these problems, the effect of alkyl substitution to improve solubility is tested with the introduction of methyl groups on nitrogen, leading to DPPM oligomers. Methyl groups are used to simulate the steric effect of longer alkyl chains as their effects on the electronic structure are very similar. The stability issues and non-planarity resulting from methyl substitution are addressed with vinyl and thienyl spacers, which lead to DPPMV and DPPM-T oligomers. Properties for oligomers with close to 24 double bonds along the backbone are compared in Table II. The absorption spectra of the same oligomers are presented in Fig. 2
DPPM oligomers are closed-shell systems up to the tetramer. Pentamer and hexamer are almost pure singlets with $\left\langle S^{2}\right\rangle$ values of 0.08 and 0.19 . Spin contamination gets severe for longer oligomers, but the $\left\langle S^{2}\right\rangle$ value reaches only 1.34 for 12-DPPM. Because DFT and CASSCF/NEVPT2 results do not differ dramatically for DPP oligomers with much larger $\left\langle S^{2}\right\rangle$ values, the calculation was all done with DFT.

DPPM oligomers are non-planar with dihedral angles between the DPPM units of $44^{\circ}$. The non-planarity reduces conjugation along the backbone and prevents the inversion of single and double bonds that was observed for DPP oligomers compared to isolated DPP (Scheme 2). Figure 3 compares the energy levels of DPP and DPPM oligomers. Methyl substitution pushes valence and conduction bands up, reducing IE $(0.5 \mathrm{eV})$ and EA $(0.6 \mathrm{eV})$. The reduced conjugation in DPPM decreases the conduction bandwidth by $0.9 \mathrm{eV}$. The bandgap and excitation energy to the strongly allowed state of 12-DPPM increase by less than $0.1 \mathrm{eV}$ compared to those of 12-DPP (Fig. 2).

To restore planarity, it is sufficient to introduce a vinyl spacer between the DPPM units to reduce the steric repulsion between the methyl and carbonyl groups. This creates donor-acceptor oligomers DPPM-V. Upon return to planarity, the single and double bond lengths along the backbone invert again (Scheme 2), and the spin

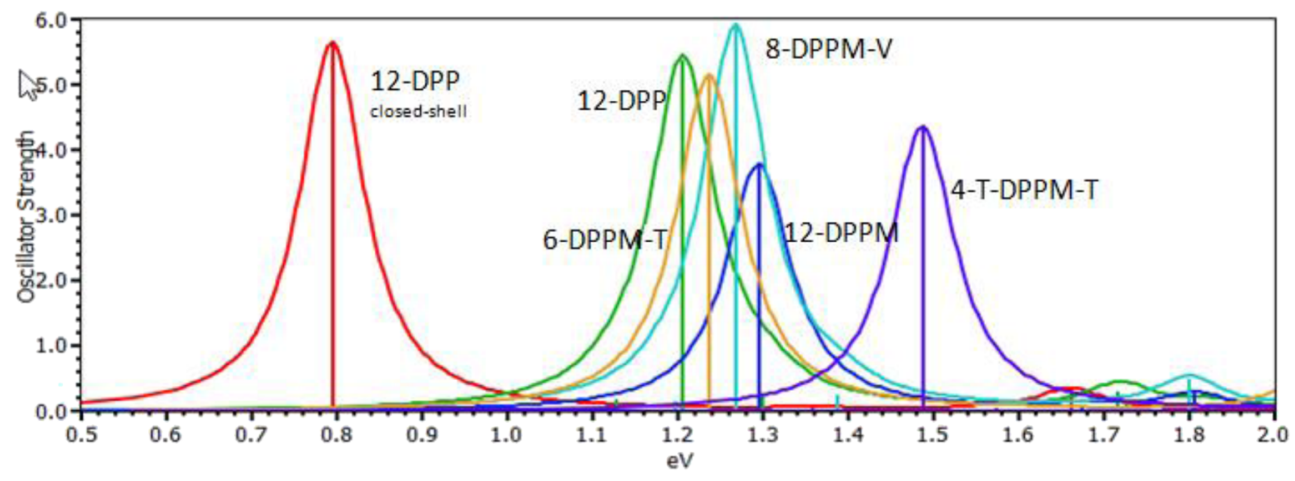

FIG. 2. TDDFT absorption spectra of closed-shells 12-DPP, 12-DPP, 12-DPPM, 8-DPPM-V, 6-DPPM-T, and 4-T-DPPM-T. 


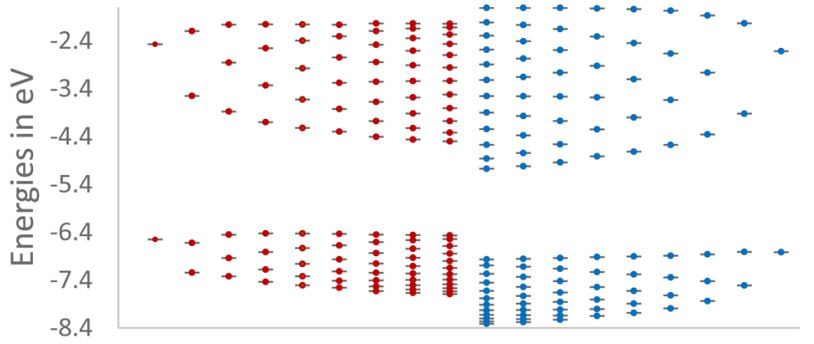

FIG. 3. Orbital energies of DPPM and DPP oligomers.

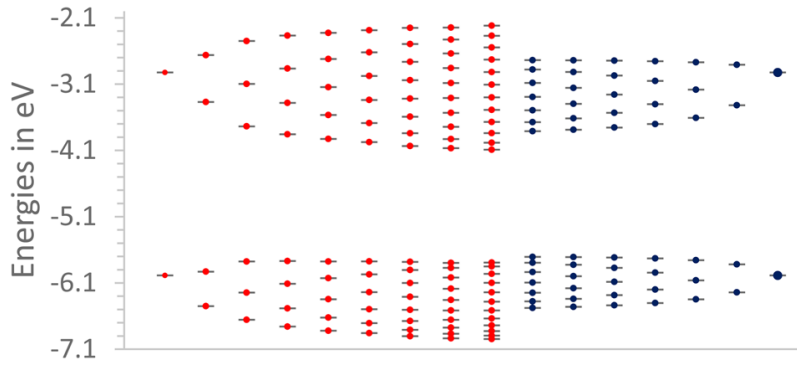

FIG. 5. Orbital energies of DPPM-T and T-DPPM-T oligomers. contamination increases due to the unpaired electrons in the terminal units. Figure 4 reveals that vinyl-spacers push the valence and conduction bands up by $0.2 \mathrm{eV}-0.3 \mathrm{eV}$ and decrease the valence and conduction bandwidths slightly but have no effect on the bandgap. Thus, although the DPPM-V oligomers are planar, the bandwidth is not increased because the system has donor-acceptor character. The absorption maximum shifts by $0.07 \mathrm{eV}$ to lower energy, and the oscillator strength increases (Fig. 2). Adding vinyl groups at the ends of the oligomers in the same fashion as with thiophene rings (Scheme 1) does not alter the energy levels as the entry for V-8-DPPM-V below that for 8-DPPM-V in Table II shows.

DPPM-T oligomers are closed-shell up to the trimer. Then, the $\left\langle\mathrm{S}^{2}\right\rangle$ value increases to 2.0 for the dodecamer. Thienyl spacers are intrinsically aromatic and resist single and double bond inversion. As a result, there is almost no bond length alternation in DPPM-T oligomers. Reducing bond length alternation is one of the strategies to reduce bandgaps in organic polymers. ${ }^{40}$ It might also be beneficial for charge carrier transport. The IEs and EAs of 12-DPPM-T are reduced by over $1 \mathrm{eV}$ compared to DPP oligomers (Table II). The EA of the polymer would be slightly above $4 \mathrm{eV}$, which is the ideal value for $n$-doping and electron transport. ${ }^{39}$ The valence bandwidth is reduced by only $\sim 0.3 \mathrm{eV}$ compared to that of 12-DPP, but the conduction bandwidth is halved. Such a bandwidth decrease is generally observed for donor-acceptor systems because interaction between orbitals is inversely proportional to the energy difference between them. DPPM-T systems have the smallest bandgaps among the oligomers studied, but the absorption maximum of 6-DPPM-T lies $0.04 \mathrm{eV}$ above that of 12-DPPM.

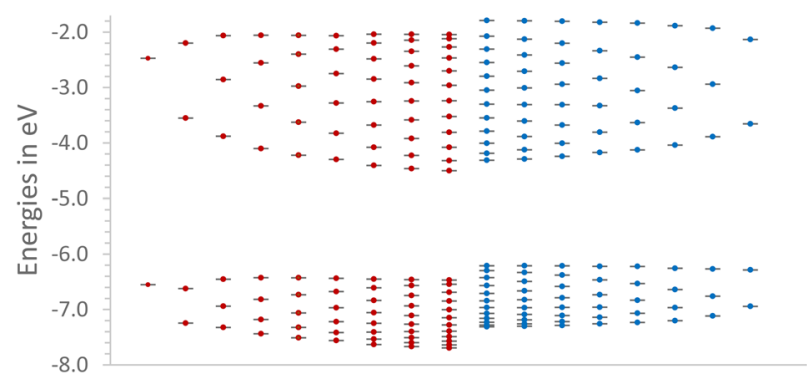

FIG. 4. Orbital energies of DPPM and DPPM-V oligomers.
Figure 5 compares the energy levels of DPPM-T and T-DPPMT oligomers. All T-DPPM-T oligomers that were investigated (up to the heptamer) are closed-shell species. The bandgap is larger than those of all other systems. The second thienyl spacer reduces the valence bandwidths of 4-T-DPPM-T compared to that of 6-DPPM$\mathrm{T}$ from $1.2 \mathrm{eV}$ to $0.8 \mathrm{eV}$ and the conduction bandwidth from $1.7 \mathrm{eV}$ to $1.1 \mathrm{eV}$. The conduction bandwidth estimated for the poly-TDPPM-T is, therefore, less than 1/3 that of poly-DPP and about half that of poly-DPPM-T. The EA would be $\sim 0.3 \mathrm{eV}$ lower than that of poly-DPPM-T. The absorption spectrum is blue-shifted compared to those of the other systems.

\section{DISCUSSION}

For the majority of copolymers used in organic electronics, the properties of the corresponding homopolymers are known as well. DPP is the exception as the original synthesis produced the DPP unit with attached phenyl groups. Different aryl groups can replace the phenyl groups, but to the best of the author's knowledge, DPP homooligomers or homopolymers have never been reported. The present theoretical investigation reveals that DPP homooligomers and all derivatives included in this study with less than two aromatic spacers are open-shell di- or poly-radicals. The reason for this radical character is that the conjugation along the backbone of DPP oligomers is extremely strong. As a consequence, the single and double bond positions switch upon the increase in the chain length, leaving an unpaired electron at each terminal unit. This electron can be bound by end-capping the $\mathrm{CH}_{2}$-groups, but the HOMOLUMO gaps are still so small that symmetry breaking occurs by HOMO-LUMO mixing. As the bandgap is 0 for the decamer and longer oligomers according to CASSCF/NEVPT2 calculations, polyDPP could be a synthetic metal. This is in contrast to other small bandgap systems, where symmetry breaking opens the bandgap. The radical nature of these systems can lead, of course, to stability issues. However, diradicals are increasingly used in organic electronics, and exceptional conductivities have been reported. ${ }^{41-43}$ Moderate diradical character has also been demonstrated to facilitate singlet fission ${ }^{4,44-48}$ and is essential for increasing nonlinear optical properties. $^{49}$

Based on the earlier work on donor-acceptor systems, ${ }^{13-16}$ the conduction bandwidth of poly-DPP was expected to be huge because poly-T-DPPM-T has a wider conduction band than 


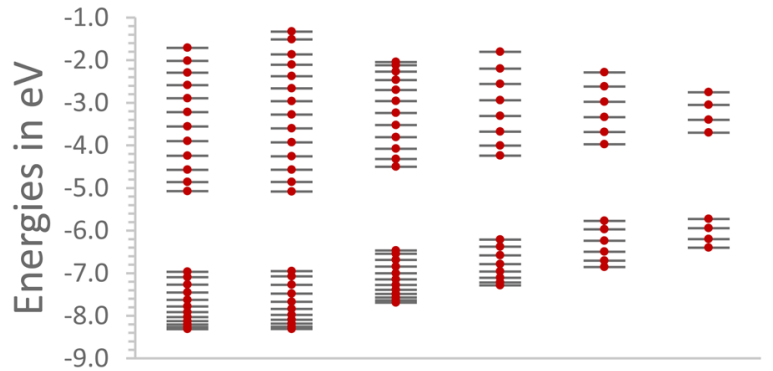

FIG. 6. Comparison of the valence and conduction bands of 12-DPP, $\mathrm{CH}_{2}$-capped 12-DPP, 12-DPPM, 8-DPPM-V, 6-DPPM-T, and 4-T-DPPM-T.

polythiophene. The comparison of the orbital energies of oligomers with 24 or very close to 24 double bonds along the backbone in Fig. 6 nicely demonstrates the effect of donor-acceptor substitution on the bandwidth. It might look like using fewer energy levels for the larger repeat units may bias the results, but it is clearly visible from Fig. 5 that not much increase in bandwidths occurs for the longer T-DPPM-T-oligomers and, therefore, also for the polymer.

Because the wavefunctions of the open-shell systems are highly spin-contaminated, multi-reference calculations were carried out in addition to DFT. Use of the CASSCF method to compare a series of molecules with different electronic structures and sizes on equal footing is not straightforward. The DPP monomer has already $12 \pi$ electrons and requires an active space of 12 electrons and 10 orbitals if all $\pi$-electrons should be included in the active space. The corresponding active space of the dimer is, therefore, already beyond the capacity of most current computers. CASSCF with the density matrix renormalization group ${ }^{54}$ approach can handle larger active spaces but requires huge amounts of disk space. The comparison of the two series, $\operatorname{CASSCF}(4,4)$ and $\operatorname{CASSCF}(2 n, 2 n)$, shows that the results agree within $0.2 \mathrm{eV}-0.3 \mathrm{eV}$ including all properties. Therefore, it is probably safe to assume that the non-dynamic correlation effects do not reach far beyond the frontier orbitals and that the calculations with both active spaces are sufficient. It is puzzling that ground state $\Delta \mathrm{E}_{\mathrm{S}-\mathrm{T}}$ values with both active spaces do not decrease monotonically as expected but produce a minimum at 4-5 DPP units and then start increasing and fluctuating. This trend is not observed when the triplet excitation energies are calculated with the same active spaces. Another disadvantage of the CASSCF/NEVPT2 method is that with state averaging, the excitation energies depend on the number of states calculated. Therefore, there is an arbitrariness in the results that makes accurate theoretical prediction of properties very difficult. It is encouraging, however, that there are no dramatic differences between the CASSCF/NEVPT2 and broken symmetry DFT results so that the use of broken symmetry DFT can be justified despite the very bad expectation values of the spin operator.

\section{CONCLUSIONS}

Homopolymers of DPP are strongly conjugated and have extremely wide low-lying conduction bands and small or zero bandgaps. Therefore, they would be stable in the n-doped form and should be very good electron conductors. Because of their small HOMO-LUMO gaps, symmetry breaking occurs, and diradical character increases with chain lengths. This might further increase conductivity but will also lead to stability issues. Since IE and EA are very high, it might be possible to stabilize the radicals with branched alkyl groups on nitrogen to prevent radical recombination.

Introduction of vinyl or thienyl groups creates donor-acceptor systems with less radical character but with smaller conduction bandwidths and lower EAs. Thus, optoelectronic properties of DPP systems can be adjusted with donor spacers from small bandgap semiconductors to almost zero bandgap systems with infrared absorption maxima.

Donor-acceptor substitution induces localization of electrons on the acceptor groups. Reducing the number of aromatic rings between the DPP units as in poly-DPPM-T compared to poly-TDPPM-T might be useful in finding a compromise between bandwidth and stability.

A comparison of CASSCF/NEVPT2 and DFT results reveals no major deviations with broken symmetry calculations despite the extreme spin contamination of the wavefunctions of the long oligomers. Therefore, using the much cheaper DFT can be justified for these systems.

\section{ACKNOWLEDGMENTS}

The author thanks TÜBITAK for financial support through Project No. $217 Z 068$.

\section{DATA AVAILABILITY}

The data that support the findings of this study are available from the corresponding author upon reasonable request.

\section{REFERENCES}

${ }^{1}$ Q. Liu, S. E. Bottle, and P. Sonar, "Developments of diketopyrrolopyrrole-dyebased organic semiconductors for a wide range of applications in electronics," Adv. Mater. 32(4), 1903882 (2020).

${ }^{2}$ X. Yan, M. Xiong, J.-T. Li, S. Zhang, Z. Ahmad, Y. Lu, Z.-Y. Wang, Z.-F. Yao, J.-Y. Wang, X. Gu, and T. Lei, "Pyrazine-flanked diketopyrrolopyrrole (DPP): A new polymer building block for high-performance n-type organic thermoelectrics," J. Am. Chem. Soc. 141(51), 20215-20221 (2019).

${ }^{3}$ T. Mukhopadhyay, B. Puttaraju, S. P. Senanayak, A. Sadhanala, R. Friend, H. A. Faber, T. D. Anthopoulos, U. Salzner, A. Meyer, and S. Patil, "Air-stable $n$-channel diketopyrrolopyrrole-diketopyrrolopyrrole oligomers for high performance ambipolar organic transistors," ACS Appl. Mater. Interfaces 8(38), 25415-25427 (2016).

${ }^{4}$ K. C. Krishnapriya, P. Roy, B. Puttaraju, U. Salzner, A. J. Musser, M. Jain, J. Dasgupta, and S. Patil, "Spin density encodes intramolecular singlet exciton fission in pentacene dimers," Nat. Commun. 10(1), 33 (2019).

${ }^{5}$ C. E. Miller, M. R. Wasielewski, and G. C. Schatz, "Modeling singlet fission in rylene and diketopyrrolopyrrole derivatives: The role of the charge transfer state in superexchange and excimer formation,” J. Phys. Chem. C 121(19), 10345-10350 (2017).

${ }^{6}$ X. Yong, G. Wu, W. Shi, Z. M. Wong, T. Deng, Q. Zhu, X. Yang, J.-S. Wang, J. Xu, and S.-W. Yang, "Theoretical search for high-performance thermoelectric donoracceptor copolymers: The role of super-exchange couplings," J. Mater. Chem. A 8(41), 21852-21861 (2020). 
${ }^{7}$ R. Lenz and O. Wallquist, "DPP chemistry-Continuous innovation," Surf. Coat. Int., Part B 85(1), 19-26 (2002).

${ }^{8}$ A. B. Tamayo, M. Tantiwiwat, B. Walker, and T.-Q. Nguyen, "Design, synthesis, and self-assembly of oligothiophene derivatives with a diketopyrrolopyrrole core," J. Phys. Chem. C 112(39), 15543-15552 (2008).

${ }^{9}$ A. B. Tamayo, B. Walker, and T.-Q. Nguyen, "A low band gap, solution processable oligothiophene with a diketopyrrolopyrrole core for use in organic solar cells," J. Phys. Chem. C 112(30), 11545-11551 (2008).

${ }^{10}$ C. Kanimozhi, N. Yaacobi-Gross, K. W. Chou, A. Amassian, T. D. Anthopoulos, and S. Patil, "Diketopyrrolopyrrole-diketopyrrolopyrrole-based conjugated copolymer for high-mobility organic field-effect transistors," J. Am. Chem. Soc. 134, 16532-16535 (2012).

${ }^{11}$ K. Tandy, G. K. Dutta, Y. Zhang, N. Venkatramaiah, M. Aljada, P. L. Burn, P. Meredith, E. B. Namdas, and S. Patil, "A new diketopyrrolopyrrole-based co-polymer for ambipolar field-effect transistors and solar cells," Org. Electron. 13(10), 1981-1988 (2012).

${ }^{12}$ E. E. Havinga, W. ten Hoeve, and H. Wynberg, "Alternate donor-acceptor small-band-gap semiconducting polymers; polysquaraines and polycroconaines," Synth. Met. 55, 299-306 (1993).

${ }^{13}$ U. Salzner, "Does the donor-acceptor concept work for designing synthetic metals? 1. Theoretical investigation of poly(3-cyano- $3^{\prime}$-hydroxybithiophene)," J. Phys. Chem. B 106, 9214-9220 (2002).

${ }^{14} \mathrm{U}$. Salzner and M. E. Köse, "Does the donor-acceptor concept work for designing synthetic metals? 2. Theoretical investigation of copolymers of 4-(dicyanomethylene-4H-cyclopenta[2,1-b:3,4- $\left.b^{\prime}\right]$ dithiophene and 3,4(ethylenedioxy)thiophene," J. Phys. Chem. B 106, 9221-9226 (2002).

${ }^{15}$ U. Salzner, O. Karaltı, and S. Durdaği, "Does the donor-acceptor concept work for designing synthetic metals? III. Theoretical investigation of copolymers between quinoid acceptors and aromatic donors," J. Mol. Model. 12, 687-701 (2006).

${ }^{16}$ U. Salzner, "Effect of donor-acceptor substitution on optoelectronic properties of conducting organic polymers," J. Chem. Theory Comput. 10(11), 4921-4937 (2014).

${ }^{17}$ J. P. Perdew, "Density functional approximation for the correlation energy of the inhomogeneous electron gas," Phys. Rev. B 33, 8822-8824 (1986).

${ }^{18}$ A. D. Becke, "Density-functional thermochemistry. III. The role of exact exchange,” J. Chem. Phys. 98, 5648-5652 (1993).

${ }^{19}$ U. Salzner, P. G. Pickup, R. A. Poirier, and J. B. Lagowski, “Accurate method for obtaining band gaps in conducting polymers using a DFT/hybrid approach," J. Phys. Chem. A 102, 2572-2578 (1998).

${ }^{20} \mathrm{U}$. Salzner and A. Aydin, "Improved prediction of properties of $\pi$-conjugated oligomers with range-separated hybrid density functionals," J. Chem. Theory Comput. 7(8), 2568-2583 (2011).

${ }^{21}$ W. J. Hehre, R. Ditchfield, and J. A. Pople, "Self-consistent molecular orbital methods. XII. Further extensions of Gaussian-type basis sets for use in molecular orbital studies of organic molecules," J. Chem. Phys. 56(5), 2257-2261 (1972).

${ }^{22}$ K. Yamaguchi, Y. Takahara, T. Fueno, and K. Nasu, "Ab initio MO calculations of effective exchange integrals between transition-metal ions via oxygen dianions: Nature of the copper-oxygen bonds and superconductivity," Jpn. J. Appl. Phys. 26(8), L1362-L1364 (1987).

${ }^{23}$ P. Pulay and T. P. Hamilton, "UHF natural orbitals for defining and starting MC-SCF calculations,” J. Chem. Phys. 88, 4926 (1988).

${ }^{24}$ S. Grimme and A. Hansen, "A practicable real-space measure and visualization of static electron-correlation effects,” Angew. Chem., Int. Ed. 54(42), 12308-12313 (2015).

${ }^{25}$ F. Neese and F. Wennmohs, ORCA 4.2.1. Manual (phpBB Forum Software, 2020), available at https://orcaforum.kofo.mpg.de/app.php/dlext/?view=detail\&df id $=80$.

${ }^{26}$ C. Angeli, R. Cimiraglia, S. Evangelisti, T. Leininger, and J.-P. Malrieu, "Introduction of $n$-electron valence states for multireference perturbation theory," J. Chem. Phys. 114(23), 10252-10264 (2001).

${ }^{27}$ C. Angeli, R. Cimiraglia, and J.-P. Malrieu, “ $n$-electron valence state perturbation theory: A spinless formulation and an efficient implementation of the strongly contracted and of the partially contracted variants," J. Chem. Phys. 117(20), 9138-9153 (2002).
${ }^{28}$ C. Angeli, M. Pastore, and R. Cimiraglia, "New perspectives in multireference perturbation theory: The $n$-electron valence state approach,” Theor. Chem. Acc. 117(5), 743-754 (2007).

${ }^{29}$ K. Kamada, K. Ohta, A. Shimizu, T. Kubo, R. Kishi, H. Takahashi, E. Botek, B. Champagne, and M. Nakano, "Singlet diradical character from experiment," J. Phys. Chem. Lett. 1(6), 937-940 (2010).

${ }^{30}$ F. Weigend, M. Kattannek, and R. Ahlrichs, "Approximated electron repulsion integrals: Cholesky decomposition versus resolution of the identity methods," J. Chem. Phys, 130(16), 164106 (2009).

${ }^{31}$ F. Neese, F. Wennmohs, A. Hansen, and U. Becker, "Efficient, approximate and parallel Hartree-Fock and hybrid DFT calculations. A 'chain-ofspheres' algorithm for the Hartree-Fock exchange," Chem. Phys. 356(1), 98-109 (2009).

${ }^{32}$ F. Weigend and R. Ahlrichs, "Balanced basis sets of split valence, triple zeta valence and quadruple zeta valence quality for $H$ to $R n$ : Design and assessment of accuracy," Phys. Chem. Chem. Phys. 7(18), 3297-3305 (2005).

${ }^{33}$ F. Weigend, "Hartree-Fock exchange fitting basis sets for $\mathrm{H}$ to Rn," J. Comput. Chem. 29(2), 167-175 (2008).

${ }^{34}$ M. J. Frisch, G. W. Trucks, H. B. Schlegel, G. E. Scuseria, M. A. Robb, J. R. Cheeseman, G. Scalmani, V. Barone, G. A. Petersson, H. Nakatsuji, X. Li, M. Caricato, A. V. Marenich, J. Bloino, B. G. Janesko, R. Gomperts, B. Mennucci, H. P. Hratchian, J. V. Ortiz, A. F. Izmaylov, J. L. Sonnenberg, D. Williams, F. Ding, F. Lipparini, F. Egidi, J. Goings, B. Peng, A. Petrone, T. Henderson, D. Ranasinghe, V. G. Zakrzewski, J. Gao, N. Rega, G. Zheng, W. Liang, M. Hada, M. Ehara, K. Toyota, R. Fukuda, J. Hasegawa, M. Ishida, T. Nakajima, Y. Honda, O. Kitao, H. Nakai, T. Vreven, K. Throssell, J. A. Montgomery, Jr., J. E. Peralta, F. Ogliaro, M. J. Bearpark, J. J. Heyd, E. N. Brothers, K. N. Kudin, V. N. Staroverov, T. A. Keith, R. Kobayashi, J. Normand, K. Raghavachari, A. P. Rendell, J. C. Burant, S. S. Iyengar, J. Tomasi, M. Cossi, J. M. Millam, M. Klene, C. Adamo, R. Cammi, J. W. Ochterski, R. L. Martin, K. Morokuma, O. Farkas, J. B. Foresman, and D. J. Fox, Gaussian 16, Rev. B.01, Wallingford, CT, 2016.

${ }^{35}$ F. Neese, “The ORCA program system," Wiley Interdiscip. Rev.: Comput. Mol. Sci. 2(1), 73-78 (2012).

${ }^{36}$ F. Neese, F. Wennmohs, U. Becker, and C. Riplinger, "The ORCA quantum chemistry program package," J. Chem. Phys. 152(22), 224108 (2020).

${ }^{37}$ J.-D. Chai, "Density functional theory with fractional orbital occupations," J. Chem. Phys. 136(15), 154104 (2012).

${ }^{38}$ L. Kronik, T. Stein, S. Refaely-Abramson, and R. Baer, "Excitation gaps of finitesized systems from optimally tuned range-separated hybrid functionals," J. Chem. Theory Comput. 8(5), 1515-1531 (2011).

${ }^{39}$ J. Dhar, U. Salzner, and S. Patil, "Trends in molecular design strategies for ambient stable n-channel organic field effect transistors," J. Mater. Chem. C 5(30), 7404-7430 (2017).

${ }^{40} \mathrm{~J}$. Roncali, "Synthetic principles for bandgap control in linear $\pi$-conjugated systems," Chem. Rev. 97(1), 173-206 (1997).

${ }^{41}$ K. Yang, X. Zhang, A. Harbuzaru, L. Wang, Y. Wang, C. Koh, H. Guo, Y. Shi, J. Chen, H. Sun, K. Feng, M. C. Ruiz Delgado, H. Y. Woo, R. P. Ortiz, and X. Guo, "Stable organic diradicals based on fused quinoidal oligothiophene imides with high electrical conductivity," J. Am. Chem. Soc. 142(9), 4329-4340 (2020).

${ }^{42}$ W. Wang, L. Ge, G. Xue, F. Miao, P. Chen, H. Chen, Y. Lin, Y. Ni, J. Xiong, $\mathrm{Y}$. $\mathrm{Hu}, \mathrm{J}$. Wu, and Y. Zheng, "Fine-tuning the diradical character of molecular systems via the heteroatom effect," Chem. Commun. 56(9), 1405-1408 (2020).

${ }^{43}$ J. D. Yuen, M. Wang, J. Fan, D. Sheberla, M. Kemei, N. Banerji, M. Scarongella, S. Valouch, T. Pho, R. Kumar, E. C. Chesnut, M. Bendikov, and F. Wudl, "Importance of unpaired electrons in organic electronics," J. Polym. Sci., Part A: Polym. Chem. 53(2), 287-293 (2015).

${ }^{44}$ T. Ullrich, P. Pinter, J. Messelberger, P. Haines, R. Kaur, M. M. Hansmann, D. Munz, and D. M. Guldi, "Singlet fission in carbene-derived diradicaloids," Angew. Chem., Int. Ed. 59(20), 7906-7914 (2020).

${ }^{45}$ T. Nagami, K. Okada, H. Miyamoto, W. Yoshida, T. Tonami, and M. Nakano, "Molecular design principle for efficient singlet fission based on diradical characters and exchange integrals: Multiple heteroatom substitution effect on anthracenes," J. Phys. Chem. C 124(22), 11800-11809 (2020). 
${ }^{46} \mathrm{~S}$. Ito and M. Nakano, "Theoretical molecular design of heteroacenes for singlet fission: Tuning the diradical character by modifying $\pi$-conjugation length and aromaticity," J. Phys. Chem. C 119(1), 148-157 (2015).

${ }^{47}$ T. Minami and M. Nakano, "Diradical character view of singlet fission," J. Phys. Chem. Lett. 3(2), 145-150 (2012).

${ }^{48}$ S. Ito, T. Minami, and M. Nakano, "Diradical character based design for singlet fission of condensed-ring systems with $4 n \pi$ electrons," J. Phys. Chem. C 116(37), 19729-19736 (2012).

${ }^{49}$ M. Nakano, R. Kishi, N. Nakagawa, S. Ohta, H. Takahashi, S.-I. Furukawa, K. Kamada, K. Ohta, B. Champagne, E. Botek, S. Yamada, and K. Yamaguchi, "Second hyperpolarizabilities $(\gamma)$ of bisimidazole and bistriazole benzenes: Diradical character, charged state, and spin state dependences," J. Phys. Chem. A 110(12), 4238-4243 (2006).

${ }^{50}$ M. Nakano, R. Kishi, S. Ohta, A. Takebe, H. Takahashi, S.-I. Furukawa, T. Kubo, Y. Morita, K. Nakasuji, K. Yamaguchi, K. Kamada, K. Ohta, B. Champagne, and E. Botek, "Origin of the enhancement of the second hyperpolarizability of singlet diradical systems with intermediate diradical character," J. Chem. Phys. 125(7), 074113 (2006).

${ }^{51}$ K. Kamada, K. Ohta, T. Kubo, A. Shimizu, Y. Morita, K. Nakasuji, R. Kishi, S. Ohta, S.-I. Furukawa, H. Takahashi, and M. Nakano, "Strong two-photon absorption of singlet diradical hydrocarbons," Angew. Chem., Int. Ed. 46(19), 3544-3546 (2007).

${ }^{52}$ M. Nakano, R. Kishi, S. Ohta, H. Takahashi, T. Kubo, K. Kamada, K. Ohta, E. Botek, and B. Champagne, "Relationship between third-order nonlinear optical properties and magnetic interactions in open-shell systems: A new paradigm for nonlinear optics," Phys. Rev. Lett. 99(3), 033001 (2007).

${ }^{53}$ M. Nakano, H. Nagai, H. Fukui, K. Yoneda, R. Kishi, H. Takahashi, A. Shimizu, T. Kubo, K. Kamada, K. Ohta, B. Champagne, and E. Botek, "Theoretical study of third-order nonlinear optical properties in square nanographenes with open-shell singlet ground states," Chem. Phys. Lett. 467(1-3), 120-125 (2008).

${ }^{54} \mathrm{~S}$. R. White, "Density matrix formulation for quantum renormalization groups," Phys. Rev. Lett. 69(19), 2863-2866 (1992). 